\title{
Modelling sea lice control by lumpfish on Atlantic salmon farms: interactions with mate limitation, temperature and treatment rules
}

\author{
Gregor F. McEwan ${ }^{1} *$, Maya L. Groner ${ }^{2,3}$, Allegra A. B. Cohen ${ }^{4}$, Albert K. D. Imsland ${ }^{5,6}$, \\ Crawford W. Revie ${ }^{1,7}$ \\ ${ }^{1}$ Department of Health Management, University of Prince Edward Island, Charlottetown, PE \\ C1A 4P3, Canada \\ ${ }^{2}$ Prince William Sound Science Center, Cordova, AK 99574, USA \\ ${ }^{3}$ US Geological Survey, Western Fisheries Research Center, Seattle, WA 98115, USA \\ ${ }^{4}$ Department of Agricultural and Biological Engineering, University of Florida, Gainesville, \\ FL 32611-0570, USA
}

${ }^{5}$ Akvaplan-niva Iceland Office, Akralind 4, 201 Kópavogur, Iceland

${ }^{6}$ Department of Biology, University of Bergen, High Technology Centre, 5020 Bergen, Norway

${ }^{7}$ Department of Computer and Information Sciences, University of Strathclyde, Glasgow G1 1QE, UK

*Corresponding author: gmcewan@upei.ca

Running header: McEwan et al.: Modelling sea lice control by lumpfish

ABSTRACT: Atlantic salmon farming is one of the largest aquaculture sectors in the world. A major impact on farm economics, fish welfare and, potentially, nearby wild salmonid populations, is the sea louse ectoparasite Lepeophtheirus salmonis. Sea louse infestations are most often controlled through application of chemicals, but in most farming regions, sea lice have evolved resistance to the small set of available chemicals. Therefore, alternative treatment methodologies are becoming more widely used. One increasingly common alternative treatment involves the co-culture of farmed salmon with cleaner fish, which prey on sea lice. However, despite their wide use, little is understood about the situations in which cleaner fish are most effective. For example, previous work suggests that a low parasite density results in sea lice finding it difficult to acquire mates, reducing fecundity and population growth. Other work suggests that environmental conditions such as temperature and external sea louse pressure have substantial impact on this mate limitation threshold and may even remove the effect entirely. We used an Agent-Based Model (ABM) to simulate cleaner fish on a salmon farm to explore interactions between sea louse mating behaviour, cleaner fish feeding rate, temperature and external sea louse pressure. We found that sea louse mating has a substantial effect on sea louse infestations under a variety of environmental conditions. Our results suggest that cleaner fish can control sea louse infestations most effectively by maintaining the population below critical density thresholds.

KEY WORDS: Salmon aquaculture $\cdot$ Lepeophtheirus salmonis $\cdot$ Salmo salar $\cdot$ Cyclopterus lumpus $\cdot$ Agent-based modelling $\cdot$ Mate limitation $\cdot$ Cleaner fish 


\section{Introduction}

Sea lice Lepeophtheirus salmonis, ectoparasites of salmonids, are a major concern for the aquaculture of Atlantic salmon Salmo salar; infestations by these copepodids can lead to economic losses on farms, and spillover of sea lice from farmed salmon can negatively impact wild salmonid populations (Costello 2009, Krkošek et al. 2011). Management of sea louse infestations is therefore a priority on salmon farms, but control can be challenging and expensive. For example, control of sea lice in central Norway was recently estimated to average $9 \%$ of farm revenues (Abolofia et al. 2017). On salmon farms, sea lice are often controlled through the use of chemical treatments, applied either as a bath or mixed with the fish feed (reviewed by Jackson et al. 2017). Use of alternative control strategies, such as physical barriers, non-chemical baths and sea louse predators, is increasing. These alternatives are popular for a variety of reasons. They are frequently less stressful to farmed salmon, have a lower environmental impact and may be more cost effective (Liu \& vanhauwaer Bjelland 2014). In addition, many chemical treatments are becoming less effective as sea lice are evolving resistance to them (Lees et al. 2008, Aaen et al. 2015, Stien et al. 2016, Powell et al. 2017). Due to the demand for alternative strategies for sea louse control, many of these methods are being used with limited quantification of efficacy or evaluation to optimise their effectiveness (Groner et al. 2013, Powell et al. 2017).

Co-culture of farmed salmon with cleaner fish, which prey upon sea lice, is an increasingly common alternative to chemical treatments on salmon farms. Lumpfish Cyclopterus lumpus and several species of wrasse (family Labridae) are now widely used to control sea lice (Blanco Gonzalez \& de Boer 2017, Powell et al. 2017). Cleaner fish may influence sea louse populations through 2 mechanisms. First, by preying upon sea lice, they reduce the numbers directly. Second, cleaner fish may prevent sea louse populations from establishing by preventing infestations from exceeding levels where mating is likely to occur (Stormoen et al. 2013, Groner et al. 2014). This second mechanism is appealing to managers; if sea louse densities are so low that mating opportunities are limited, exponential population growth cannot occur and fewer chemical treatments will be required (Stormoen et al. 2013, Groner et al. 2014). In contrast, when sea lice are not experiencing mate limitation, high fecundity and short generation times can lead to rapid exponential population growth, which can be challenging to control (Groner et al. 2014). While the perception is that cleaner fish are highly effective at controlling sea lice, the context dependency of these mechanisms on environmental conditions and management strategies is unknown.

Recent empirical and theoretical work on critical thresholds, or tipping points, for parasite establishment have found that they vary depending upon environmental conditions (e.g. Lloyd-Smith et al. 2005, Dallas et al. 2018). For example, population matrix projections have shown that the effect of mate limitation on population growth decreases with increasing temperature (Groner et al. 2014). In addition, it has been hypothesised that high levels of external recruitment of sea lice to a farm can swamp the effects of mate limitation (Aldrin et al. 2017). Thus, the efficacy of leveraging mate limitation for sea louse control may depend on external conditions: different environmental conditions may have different tipping points.

In this paper, we used an Agent-Based Model (ABM) to explore the interaction of lumpfish and mate limitation on sea louse population dynamics under different environmental scenarios. We selected the ABM methodology as its focus on modelling individuals is amenable to simulating mate limitation. For example, in contrast to a differential equation- 
based model where there would be a statistical distribution to reflect mating, we have explicit rules about mating in our ABM, i.e. sea louse agents have to be on the same host salmon. We used the $A B M$ to (1) quantify the synergistic role of mate limitation and lumpfish in control of sea lice. We also used it to explore the effect of key environmental and policy conditions on this synergistic interaction and on the effectiveness of lumpfish for sea lice control, namely (2) external recruitment of sea lice, (3) seawater temperature and (4) chemical treatment threshold.

The aquaculture system that we modelled is a single Atlantic salmon farm. Salmon farms are normally stocked in the spring or fall as smolts and harvested when they grow to market size (about $1.5 \mathrm{yr}$ ). The surrounding seawater flows through the cages, bringing a number of advantages, including moving water, and disadvantages, such as sea lice. The predominant sea louse species in the northern hemisphere and the one we have modelled here, L. salmonis, is found almost exclusively on salmon and trout. The sea lice are free-living as larvae, and moult into an infective stage (a copepodid) that must attach to a salmonid host to survive. While feeding upon the mucus and occasionally the blood of the host, they develop into chalimus, pre-adult and adult stages (reviewed by Costello 2006). Adult males compete to mate with females that are on the same host. Typically, they guard pre-adult females so that they can mate with them after moulting (reviewed by Costello 2006). If pre-adult females are not available, they may mate with adult females as well. Adult females produce 2 egg strings at a time, which remain attached until the eggs hatch. Females that have not mated will still produce egg strings, but the eggs are not viable.

We chose to focus on lumpfish as a mechanism for sea louse population control in this model because use of this species of cleaner fish is expanding rapidly. This is due to improving aquaculture methods for lumpfish production, lack of winter dormancy in lumpfish and rapid development (they take about 1/3 the time of other cleaner fish to reach a suitable size for stocking in aquaculture net pens, Powell et al. 2017). Indeed, between 2007 and 2016, the estimated number of lumpfish used in salmon aquaculture in Norway grew more than 15fold, from about 1 million to 15 million (Powell et al. 2017). Despite the acceleration in use of this cleaner fish, many knowledge gaps exist. Perhaps the most outstanding gaps concern, first, the development of a captive breeding program to reduce pressure on wild stocks which currently provide all gametes for production, and second, knowledge of the factors that lead to large variability in the feeding rates of lumpfish on sea lice (Powell et al. 2017). Lumpfish are generalist feeders and, on salmon farms, consume free-swimming zooplankton, biofouling on nets, salmon food pellets and sea lice (Imsland et al. 2014, 2015). Sea lice frequently constitute less than 10 to $40 \%$ of their diet (Imsland et al. 2015). Their feeding preferences depend heavily upon their genetic background, food availability and previous exposure to sea lice (Imsland et al. 2015, 2016). For example, in a recent experiment, Imsland et al. (2016) found that some genetic families of lumpfish do not eat sea lice at all, while others can effectively limit sea louse populations due to their preferential consumption of sea lice. When they do eat sea lice, they have a clear preference for the latter stages of sea louse development, e.g. the adult stages, especially adult females with egg strings (Imsland et al. 2014, 2018).

Lumpfish do not breed in net pens and, currently, the practice is to lethally harvest them at the end of a salmonid production cycle. Their natural distribution covers both sides of the North Atlantic Ocean. 


\section{Model}

For this study, we modified an ABM that we have used for previous studies to explore sea louse infestations on Atlantic salmon farms (McEwan et al. 2015, 2016). In this section, we describe how this model has been adapted for this study. Model components that have not been modified from previous papers will not be discussed in detail. Readers are referred to the relevant papers for details.

We implemented the model using AnyLogic simulation software (www.anylogic.com). Our validation was based on techniques described by Sargent (2011). Specifically, we used the following techniques:

- animation-observing the processes in progress

- degenerate tests and extreme condition tests_-verifying the model operates at the limits

- face validity — expert feedback, including processes, logic and input/output

- internal validity — checking for low stochastic variability in the model

- traces - tracking individual agents through the system

We had 2 advantages: first, extending a mature model that had been used in a number of previous studies, and second, having access to expertise on the topics of salmon aquaculture, sea lice and lumpfish.

We use the Overview, Design concepts and Details (ODD) protocol (Grimm et al. 2006) to describe the model. In Overview, we introduce the types of agents used in this model and their behaviour. In Design concepts, we describe the design principles behind the model. In Details, we provide in-depth information about how the model operates.

\subsection{Overview}

2.1.1. Purpose. The purpose of the model was to investigate the use of lumpfish to control sea lice on an Atlantic salmon farm. Specifically, we explored interactions between: (1) the rates that lumpfish feed on sea lice, (2) temperature (which alters sea louse growth), (3) treatment thresholds (which specify the level of infestation required to apply the chemical treatment), (4) the rate that sea lice migrate onto a farm (henceforth called external sea lice pressure) and (5) mate limitation in sea lice (Stormoen et al. 2013).

For all of the scenarios examined, our output measurements were sea louse abundance over time and the number of chemical treatments required during a production cycle.

2.1.2. State variables and scales. There were 3 types of agents in the model: sea louse agents, salmon agents and lumpfish agents. To avoid confusion, we refer to model entities as 'agents' in this document, e.g. modelled sea lice are 'sea louse agents', while real sea lice are 'sea lice'.

Sea louse agents and salmon agents had similar behaviours to those described in our earlier study (McEwan et al. 2015), with 3 substantial differences. First, there was a farmed population of 5000 salmon, but no wild salmon population; all salmon agents existed on a farm. Second, the sea louse agents did not evolve resistance to treatments. Third, sea louse agent mating behaviour could be optionally 'turned off' so that females automatically became 
gravid after they had reached the mature stage. These differences were introduced to allow us to address the specific research questions of this paper.

Our simulated farms had only 5000 salmon agents. Commercial salmon farms vary considerably but have grown in size over the past decade. For example, in Scotland, even small farms will tend to stock at least 100000 salmon smolts while larger farms will often be stocked with over 1 million fish (Ellis et al. 2016). In Norway, farms can hold up to 4 million fish, but most often hold between 1600000 and 3200000 (M. Stormoen pers. comm.).

We added lumpfish agents to this implementation of the model. Initial lumpfish agent numbers were $10 \%$ of salmon agent numbers, i.e. 500. Lumpfish agents consumed parasitic sea louse agents at a pre-defined rate throughout the simulation.

In this study, we ran each scenario for a single typical 18 mo production cycle, from early June until December of the subsequent year.

2.1.3. Process overview and scheduling. As with our earlier versions of the model, the salmon agents were stocked on the farm with no sea louse agents on them. Again, we simulated a temperature-dependent external pressure of free-living juvenile sea louse agents that infest the farm. Yearly temperature variation followed a sine curve, and we assumed salinity to be that of normal seawater (34 psu) and thus not detrimental to sea louse life expectancy or any other modelled ecological processes. Chemical treatments were applied when the average number of adult female lice per salmon host reached a predefined threshold that was surpassed during weekly sea lice censuses. The treatment thresholds were different depending on scenario.

Lumpfish agents were added to the farm model at the same time as the salmon agents. They were assumed to live for the full production cycle and feed on sea louse agents at a rate that was dependent on the sea louse agent population size. As lumpfish are opportunist feeders and sea lice are not the main part of their diet, we did not expect a typical functional response. Salmon agents also lived for the full production cycle, while sea louse agents experienced multiple generations with births and deaths.

\subsection{Design concepts} ABM.

In this section we describe the concepts that drove the implementation and use of our

2.2.1. Model emergence. We specified the mechanics and interactions of the individual entities in the model - sea louse agents, salmon agents and lumpfish agents. We started each experiment with 5000 salmon agents, 500 lumpfish agents and no sea louse agents. The 1:10 stocking ratio of lumpfish to salmon was intermediate relative to experimental studies which range from 4 to $15 \%$ (Imsland et al. 2014, 2018) and anecdotal reports from industry of 8 to 10\% (Brooker et al. 2018). Sea louse agent numbers were highly dynamic - due to external sea lice pressure, reproduction, mortality, predation and chemical treatments - often reaching as many as 1000 000. In this study, we recorded the number of sea louse agents at each life stage over time, and the number of chemical treatments that were applied based on the treatment threshold rules of the scenario.

2.2.2. Agent sensing. Sea louse agents sensed the current seawater temperature on the farm, which affected their development. When they were in the infectious copepodid 
stage, they selected a random salmon agent from the farm population to infest (with a userdefined probability of successful attachment). When they were reproductive, male sea louse agents were aware of female sea louse agents on the same host and they searched for virgin adult females to mate with. If none were present, they searched for available pre-adult females and guarded them until they matured and were ready to mate.

When feeding, lumpfish agents selected a random salmon agent from the total population at a frequency based on their feeding rate. Once they had selected a salmon agent to feed from, they were aware of all the sea louse agents on that host and chose the largest to eat. Size increased with developmental stage. Within a developmental stage, females were larger than males.

2.2.3. Stochasticity. Rates of sea louse agent development, mortality, attachment and dispersion were all randomly sampled from distributions determined experimentally. The sources for these distributions have been described by McEwan et al. (2015). Only development was temperature-dependent.

Lumpfish agents had a feeding rate parameter, which defined the average number of times that they fed per day. The frequency of feeding events was randomly selected from an exponential distribution.

2.2.4. Observation. In this study, we observed the abundance and developmental stages of sea louse agents and the number of chemical treatments required during a production cycle.

\subsection{Details}

2.3.1. Initialisation. In each simulation, the farm model was initialised with 5000 salmon agents, either 500 or 0 lumpfish agents, depending upon the simulation, and no sea louse agents. The model started in early June (Day 154 in the temperature cycle).

2.3.2. Input. There were several inputs that drove the dynamics of the model: seasonal temperature variation; stocking and harvesting on the farm; the external flow of sea louse agents into the farm; and application of chemical treatments to control the parasitic stages of the sea louse agents.

We modelled the temperature using a modified sine function (Groner et al. 2014):

$$
\text { day_temperature }=\text { amplitude }\left[\sin \left(\frac{\pi(\text { day }+ \text { offset })}{365}\right)\right]^{2}+\text { minimum }
$$

We created 5 temperature profiles based on typical seawater temperatures found in 2 real world locations: first, southern Norway, where very positive results have been obtained with cleaner fish (Imsland et al. 2014, Mortenson et al. 2017); and second, Scotland, where the use of cleaner fish is currently increasing (Treasurer et al. 2018). From southern Norway, we created 4 profiles based on publicly available data from the Barents Watch database (www.barentswatch.no/en/fishhealth/). Three of the profiles represent typical cool, average and warm sites. The fourth, labelled 'extra warm', was used to explore situations that are becoming more common with ocean warming (Wong et al. 2014). The Scotland profile differs from the Norway profiles in that it has less variation between the winter and summer temperatures. The parameters for all 5 profiles are listed in Table 4. 
Each day we calculated an external flow of sea louse agents into the farm. We modelled 2 sources of external sea louse agents, the first from other nearby farms, and the second a 'background' source from the environment. We assumed that nearby farms had similar conditions, including treatment regime, and so modelled their contribution to external pressure as an average of this farm's internal hatching numbers over the last $30 \mathrm{~d}$, multiplied by a constant. The constant represents both the number and distance of nearby farms. The background component of the external pressure is proportional to the number of salmon agents and the current temperature. The total number of nauplii that arrived on the farm each day is the sum of these 2 sources, as expressed by the following equation:

$$
\text { nauplii }_{\text {ext }}=C \cdot \frac{1}{30} \sum_{t=-1}^{-30} \text { hatched }_{t}+0.3\left(\frac{\text { temp }}{10}\right) . \# \text { salmon_agents }
$$

We also varied the constant $C$ that scales external lice from other farms in Eq. (2), to explore the effects of different levels of external pressure in our experiments.

Treatments were applied when a threshold count of sea louse agents was reached. In all experiments in this study, the relevant threshold was the number of adult female agents per salmon agent, though the actual threshold value varied. Sea louse agents were counted every $7 \mathrm{~d}$. If the specified threshold was exceeded, a chemical bath treatment was applied, resulting in an instant reduction of the sea louse agent population. The treatment efficacy was $95 \%$ for all parasitic stages. While high, this efficacy is average for a chemical treatment applied to naïve sea lice (e.g. Myhre Jensen et al. 2017). Such a level of efficacy would not last for long as the sea lice would develop resistance (explored by McEwan et al. 2016), but in this study, each simulation was only run for a single production cycle.

2.3.3. Submodels. There were 4 submodels running simultaneously: (1) the sea louse agent lifecycle; (2) sea louse agent mating and reproduction; (3) male sea louse agent dispersion; and (4) lumpfish agent feeding. The first 3 submodels have been described in detail by McEwan et al. (2015).

While sea louse agent mating retained the same mechanism as the previous version of our model, we made 1 extension. Here, we allowed for scenarios to be run where reproduction can occur without mating. Instead, when a female sea louse agent reached adulthood, she was assumed to have mated and was considered to be gravid. This situation reflects an assumption made in many sea louse population models (Revie et al. 2005, Rittenhouse et al. 2016). The option allowed us to explore the influence of lumpfish and chemical controls in conjunction with mate limitation (Krkošek et al. 2012, Stormoen et al. 2013, Groner et al. 2014, Cox et al. 2017).

Lumpfish agents were added when the cage was stocked (Day 1) and remained until harvest time. The number of lumpfish agents was $10 \%$ of the number of salmon agents. Lumpfish agents fed at a rate specified by the scenario. Lumpfish agent was defined in a state transition diagram (Fig. 1) in the model. They started in a resting state and progressed to the hungry state at a time sampled from an exponential distribution where the average was the feeding rate. For example, if the feeding rate was 0.25 , then they would search for sea louse agents on average 0.25 times $\mathrm{d}^{-1}$, or once every $4 \mathrm{~d}$. Once in the hungry state, they randomly picked a salmon agent on the farm and searched it for a pre-adult or adult sea louse agent to eat. They selected a sea louse agent on the salmon agent with the following preference order: gravid female, non-gravid adult female, adult male, then pre-adult, which reflects the size 
preferences of lumpfish (Imsland et al. 2014). After feeding, they returned to their resting state.

If there were no suitable sea louse agents on the selected salmon agent, the lumpfish agent waited $10 \mathrm{~min}$ and then selected another salmon agent at random. If they searched 10 salmon agents without finding any adult or pre-adult sea louse agents, they stopped searching and we assumed they fed from another source such as salmon pellets or other organisms found in most salmon pens (Imsland et al. 2015). There is no empirical information about lumpfish behaviour at the detail required for this part of the model so we estimated that the waiting time is $10 \mathrm{~min}$ and that they search 10 salmon agents. Variation reflecting uncertainty in this process comes from sampling an exponential distribution to determine the time to start searching.

\subsection{Simulation experiments}

We ran 4 simulation experiments to gain an insight into how lumpfish interact with a variety of other factors to control sea louse abundance. In the first, we determined whether the mating requirement had a substantial effect under our assumptions. In the remaining experiments, we explored how different conditions influenced the efficacy of lumpfish at reducing the number of chemical treatments required for control: external pressure, temperature and threshold for chemical treatment. For all experiments, we varied the rate of lumpfish feeding to show the impact of selecting lumpfish with different preferences for consuming sea lice. While we could have used additional lumpfish influence parameters such as number of lumpfish, we only used the single feeding rate parameter. This was to characterise feeding despite the large variability in feeding rates between lumpfish stocks due to many influences. As described in section 2.1.3., we did not expect a standard functional response.

In the experiments where temperature was not varied (Expts 1, 2 and 4), we used the Norway average profile. The parameters that remained the same for all experiments are listed in Table 1. Each scenario was replicated 50 times to capture the variability that will inevitably occur due to random processes within the model.

2.4.1. Expt 1: Requirement to mate. The purpose of this experiment was to explicitly compare modelling mating with assuming automatic reproduction. While mating is always necessary in nature, it is important to explore how assumptions about mating influence model outcomes and management decisions.

In automatic reproduction, egg production is based on the number of adult female sea louse agents (AF). We also wanted to explore how different levels of lumpfish feeding and external pressure interacted with the mating requirement. In total, there were 18 scenarios in this experiment: 2 mating requirement options (yes/no); 3 lumpfish feeding rates (none, 0.1 , 0.2 sea lice $\left.\mathrm{d}^{-1}\right)$; and 3 external pressure coefficient values $(1,2,4)$, which roughly correspond to $50 \%, 66 \%$ and $80 \%$ of sea lice coming from an external source. In all scenarios, we used the Norway average temperature profile, and set the treatment threshold at $0.5 \mathrm{AF}$ per salmon. The parameters for Expt 1 are listed in Table 2.

2.4.2. Expt 2: Varying levels of external pressure. The purpose of this experiment was to explore the efficacy of lumpfish with increasing external pressure. The efficacy of 
many treatments is highly sensitive to external pressure (Groner et al. 2013), which varies with spatial and temporal changes in oceanographic conditions (Cantrell et al. 2018).

There were 9 scenarios in this experiment: 3 lumpfish feeding rates (none, 0.1, 0.2 sea lice $\left.\mathrm{d}^{-1}\right)$; and 3 external pressure coefficient values $(4,9,19)$, which roughly correspond to $80 \%, 90 \%$ or $95 \%$ of sea lice coming from an external source. In all scenarios, we used the Norway average temperature profile, set the treatment threshold at $0.5 \mathrm{AF}$ per salmon and required explicit mating. The parameters for Expt 2 are listed in Table 3.

2.4.3. Expt 3: Different seawater temperature profiles. In the experiments up to this point, a single simulated temperature profile was used. In this experiment, we explored how variations in seawater temperature interacted with levels of lumpfish feeding to alter the levels of chemical treatment required. In all scenarios, the external sea louse pressure was set to be 10 times that of the internal re-infestation pressure $(C=10)$, and it was assumed that mating was required for a sea louse to become reproductive. One additional level of lumpfish feeding effectiveness $\left(0.3\right.$ sea lice $\left.\mathrm{d}^{-1}\right)$ was also introduced. There were 20 scenarios in this experiment: 5 temperature profiles (Scotland, Norway cool, Norway average, Norway warm, Norway extra warm); and 4 feeding rates (none, $0.1,0.2,0.3$ sea lice $\mathrm{d}^{-1}$ ). The parameters for Expt 3 are listed in Table 4.

2.4.4. Expt 4: Different treatment threshold scenarios. In all other experiments it was assumed that a common treatment threshold - 0.5 AF per salmon-was in place. This experiment explored how variations in different treatment thresholds interacted with levels of lumpfish feeding to alter the levels of chemical treatment. In all scenarios, the external sea louse pressure was set to be 10 times that of the internal re-infestation pressure $(C=10)$, and it was assumed that mating was required for a sea louse to become reproductive. As with Expt 3,1 additional level of lumpfish feeding effectiveness $\left(0.3\right.$ sea lice $\left.\mathrm{d}^{-1}\right)$ was used in this final set of experiments. There were 12 scenarios in this experiment: 3 chemical treatment thresholds $\left(0.1,0.5,1\right.$ AF per salmon) and 4 feeding rates (none, 0.1, 0.2, 0.3 sea lice $\mathrm{d}^{-1}$ ). The parameters for Expt 4 are listed in Table 5.

\section{Results}

\subsection{Expt 1: Requirement to mate}

Introducing the requirement that a female sea louse agent must find a mate before she becomes gravid resulted in substantial differences in the main outcome of interest in this set of experiments: the overall number of treatments required over a production cycle (Fig. 2). In the scenarios where lumpfish agents were feeding at a rate of 0.2 sea lice $\mathrm{d}^{-1}$, it was not necessary to apply chemical treatments when mating was required. However, without imposing this requirement (i.e. assuming that female sea louse agents could 'automatically' become gravid), 6 to 8 treatments were necessary as the level of external sea louse agent pressure increased. While some treatment was required as external pressure increased for the case where lumpfish agents were only feeding at a rate of 0.1 sea lice $\mathrm{d}^{-1}$, a similar increase in the numbers of treatments required was seen for the scenarios that did not require mating. Finally, in the cases where lumpfish agents were considered to be ineffective, the overall number of treatments increased. Once again, around twice as many were required for the scenarios that did not require mating to take place between male and female sea louse agents. 


\subsection{Expt 2: Varying levels of external pressure}

While we explored some variation in external infestation pressure in the initial set of experiments, the maximum level was only 4 times that of the internal pressure $(C=4$, Eq. 2$)$. In certain settings, it has been estimated that up to $96 \%$ of a farm's infestation pressure will be from outside that site (Aldrin et al. 2017). The second set of experiments evaluated the impact of lumpfish agent feeding rates under more elevated levels of external infestation pressure (Fig. 3). The case in which external infestation pressure was set to be 4 times that of the internal pressure (external accounts for $80 \%$ of total infestation pressure) is shown in the top panel of Fig. 3. As can be seen, when either of the 2 lumpfish agent feeding rates were assumed, the sea louse agent population of the farm over the first year was largely controlled by these cleaner fish agents. However, between 3 and 4 treatments were required in the second year of production when a feeding rate of 0.1 lice $\mathrm{d}^{-1}$ was assumed. In the absence of lumpfish agent feeding, around 6 treatments were required to keep the number of sea louse agents below the threshold level. When the external infestation pressure was raised to 9 times that of the internal level (representing $90 \%$ of the total pressure; middle panel of Fig. 3) the sea louse agents were less well controlled, particularly at the lower lumpfish agent feeding rate. This was particularly evident during the second year of production where approximately 6 treatments needed to be applied to maintain numbers below the threshold level. However, when the higher feeding rate of 0.2 sea lice $\mathrm{d}^{-1}$ was maintained by the lumpfish agents, there was no need to apply any chemical treatments.

In the final scenario, the external pressure was increased to $95 \%$ of the total infestation pressure $(C=19$, Eq. 2). In this circumstance, the sea louse agent population can get out of control relatively quickly during the warmer months of the year, particularly in the absence of more effective lumpfish agent feeding. In the absence of lumpfish agents, it was necessary to treat approximately 10 times per production cycle to maintain threshold levels, while the presence of lumpfish agents feeding at a moderate rate $\left(0.1\right.$ lice $\left.\mathrm{d}^{-1}\right)$ reduced the number of required treatments to an average of 8 per production cycle. A higher lumpfish agent feeding rate $\left(0.2\right.$ sea lice $\left.\mathrm{d}^{-1}\right)$ led to much better overall control, with an average of just over 1 treatment during the production cycle.

\subsection{Expt 3: Different seawater temperature profiles}

Seawater temperature profiles ranging from relatively 'cool' (for the North Atlantic) to fairly 'warm' were explored in conjunction with differing lumpfish feeding assumptions (Fig. 4). There was clear evidence, as would be expected, that increased water temperatures led to the need for additional treatments to maintain control. In the cases with no effective lumpfish agent feeding, the numbers of treatments rose in a fairly linear fashion from around 4 to as high as 14, in the coolest to warmest setting, respectively. A similar pattern was seen at the lowest lumpfish agent feeding rate $\left(0.1\right.$ sea lice $\left.\mathrm{d}^{-1}\right)$, though in this case, the lumpfish agents were able to control the sea louse agents in the Scottish and coolest Norwegian scenarios, while up to around 12 treatments were needed under the 'extra warm' Norway conditions. As the feeding rates of the lumpfish agents were increased, so the numbers of treatments decreased. Indeed, no treatments were required for the most effective feeding rate ( 0.3 sea lice $\mathrm{d}^{-1}$ ) except in the extra warm Norwegian setting, and here the number of treatments varied significantly across different simulation runs with a median of only around 1 treatment. 


\subsection{Expt 4: Different treatment threshold scenarios}

The final experiment explored 3 different thresholds at which treatment intervention would be mandated, in relation to the differing lumpfish feeding rates (Fig. 5). When no effective lumpfish agent control was simulated, around 8 treatments were required over a production cycle. Contrary to what might be expected, the lower threshold level $(0.1 \mathrm{AF}$ per salmon) did not result in an overall increase in the number of treatments over a production cycle. This may imply that a higher overall level of control led to no additional treatments despite the fact that intervention was required at a much earlier point. This was even more apparent for the case where a lumpfish agent feeding rate of 0.1 sea lice $\mathrm{d}^{-1}$ was assumed, with the number of treatments per production cycle falling from around 7-8 to just 4 when the strictest treatment threshold level was adopted. For the more effective lumpfish agent feeding rates, no treatments were required when a threshold was set to $0.5 \mathrm{AF}$ per salmon or above. There was evidence of treatments being administered in some of the simulation runs when the feeding rate was 0.2 sea lice $\mathrm{d}^{-1}$ and the threshold was set to $0.1 \mathrm{AF}$ per salmon.

\section{Discussion}

Control of infectious marine diseases can be challenging and expensive (Groner et al. 2016a). Our categorical analyses suggest that there are 'tipping points' below which disease is less likely to invade or at which management requires fewer interventions. Our results suggest that, for sea louse infestations of farmed Atlantic salmon, management of sea lice to low levels (e.g. 0.1 AF per salmon) results in fewer parasites with comparable levels of effort (i.e. treatments) required. This occurs because, at low densities, sea lice are less likely to find a mate on the same host. Indeed, running scenarios where adult females are not required to find a mate in order to reproduce indicates that this tipping point disappears when mate limitation is not modelled. While this is not a realistic scenario, it aids in quantifying the effect of mate limitation on sea louse population growth. Moreover, the benefit of mate limitation appears to be robust to a wide range of external infestation pressures. Combining low levels of treatment threshold with the use of cleaner fish, which preferentially target adult sea lice, can dramatically reduce the number of treatments. These results provide motivation for future investigation into defining the tipping points in this system.

Previous studies of mate limitation in sea lice have considered mechanisms such as mate availability during reproduction and the role of parasite aggregation. For example, Krkošek et al. (2012), Stormoen et al. (2013), Groner et al. (2014) and Cox et al. (2017) all used different methods to come to similar conclusions about when mate limitation occurs. However, they did not take into account many of the strategies that sea lice have evolved to overcome negative effects of mate limitation. For instance, sea lice can store multiple spermatophores from different males and use them to inseminate egg strings when no male is available to mate with (Todd et al. 2005). Moreover, males can switch hosts when they determine that competition with other males is too high or there are few or no females on their host (Connors et al. 2011, Stephenson 2012). One of the benefits of agent-based modelling is that 'sensing' and 'memory' can be used to make contextual changes amongst individuals. Thus, modelling sperm storage or male dispersal as a function of local conditions (i.e. the population of sea lice on 1 host) is easier to implement than would be the case in more traditional modelling approaches. While we did not examine higher treatment thresholds, previous studies suggest that mate limitation disappears at abundances of around 2 
adult females per host (Krkošek et al. 2012, Stormoen et al. 2013, Groner et al. 2014). In our study, which accounts for spermatophore storage and host switching by male sea lice, it is likely that mate limitation would disappear at higher densities of sea lice. Unfortunately, one of the trade-offs with the use of ABMs is that scenarios which generate such high numbers of agents are, currently, prohibitively slow to run.

Our model demonstrates how critical mating behaviour is during initial infestation stages and when mean infection intensities are low. Many models of sea louse dynamics on farms do not account for mate limitation in sea lice, and make the simplifying assumption that all females have reproductive success (Revie et al. 2005, Groner et al. 2013, Rittenhouse et al. 2016). While these assumptions may have been appropriate for the scenarios modelled in those studies, where mean sea louse abundances would frequently exceed 2 adult females per salmon, they are not appropriate for modelling recent operations within some salmon farming settings, for example in Norway or British Columbia, where overall numbers of adult females are typically controlled to less than a single sea louse on most fish (Stormoen et al. 2013).

Due to a lack of information, our model of lumpfish feeding behaviours on sea lice is relatively simplistic, with constant feeding rates over time and no mortality of lumpfish. Recent studies suggest that feeding rates of lumpfish on sea lice can vary substantially both temporally (Imsland et al. 2014) and among genetic strains (Imsland et al. 2016), though the mechanisms for this variation are unclear. While it has not yet been quantified, temperature has anecdotally been observed to influence lumpfish feeding, with higher rates occurring at lower temperatures (M. Stormoen pers. obs.). As discussed earlier (see Section 1.1.), lumpfish are opportunistic feeders and the presence of other pelagic food sources likely influences their rate of consumption of sea lice (Powell et al. 2017). Our model simplifications may ignore some important synergistic and antagonistic effects. For example, at low temperatures, increased lumpfish feeding and decreased sea louse reproduction and growth may result in a significantly greater impact of the lumpfish. As data on these behaviours become available, models may be refined to incorporate them, which will improve estimates of lumpfish control and context-dependency of lumpfish behaviour. Nonetheless, this simplistic model is useful conceptually and can be further adapted as new information becomes available.

Over the past $6 \mathrm{yr}$, lumpfish have become the most commonly used cleaner fish on salmon farms, being stocked on many farms in the eastern Atlantic (Powell et al. 2017). Therefore, further quantification of population dynamics and feeding behaviours should be a research priority. Powell et al. (2017) highlighted several concerns regarding the use of lumpfish to control sea lice including (1) the need to quantify optimal stocking densities and (2), with the advent of lumpfish aquaculture, the need to select for genetic lines that preferentially consume sea lice. Our models suggest that sea lice can be effectively controlled with a stocking density of 1 lumpfish for every 10 salmon, so long as the lumpfish are feeding at a minimum rate of 0.3 sea lice $\mathrm{d}^{-1}$. Some genetic stocks of lumpfish appear to have even higher rates of sea louse consumption (Imsland et al. 2016). If lumpfish breeding can consistently select for this trait, lower densities of lumpfish may be sufficient.

Our study has also illustrated some potentially important interactions between lumpfish stocking, environmental conditions and use of chemical treatments. For example, as seawater temperatures rise, the level of effectiveness and/or stocking density of lumpfish will also have to increase in order to maintain similar control of sea lice. In the higher seawater temperature scenarios we explored, there was also evidence of increased variability in the 
estimated number of treatment interventions required. This stochasticity may be indicative of the fact that the lumpfish feeding rates were close to a tipping point. This point appears to be borne out by the outcomes seen for the most effective feeding rate $\left(0.3\right.$ sea lice $\left.\mathrm{d}^{-1}\right)$, where the model indicated a definite, but highly variable, need for treatments at the highest seawater temperature profile. This represents potential future conditions if sea surface temperatures continue to increase. Indeed, previous models have shown a very high sensitivity of sea lice to seawater temperature, which controls developmental rates (Groner et al. 2014, 2016b). Similarly, some aspects of lumpfish management interact with regulatory policy. In general, reducing the threshold level at which treatments are mandated, perhaps contrary to intuition, did not lead to an increase in the number of treatments. The 1 exception was for the lowest threshold explored ( $0.1 \mathrm{AF}$ per salmon) at a modest level of lumpfish feeding, where additional, likely 'unnecessary', treatments were indicated. This suggests that sites which have historically demonstrated sound control using lumpfish should be given some latitude as to treatment threshold levels to avoid the use of needless chemical interventions.

\section{Conclusion}

Previous research on sea louse control has focused on identifying thresholds and highly sensitive gradients in host density and environmental conditions such as temperature and salinity that influence sea louse population dynamics (Lloyd-Smith et al. 2005, Krkošek et al. 2009, Groner et al. 2016b). Infectious disease is, however, an interaction between the host, environment and pathogen, and our research demonstrates that parasite-density thresholds also influence infestation success. From a management perspective, controlling parasite densities to keep them below identified tipping points may be preferable to manipulating other previously identified tipping points such as host densities. Given the urgent need to control sea lice, the use of lumpfish has preceded the majority of research about the effectiveness of this fish (Imsland et al. 2014, 2015, 2016, Powell et al. 2017). Our results confirm those of other studies that suggest lumpfish can be effective at controlling sea lice, particularly when lumpfish stocking is combined with low density thresholds for chemical treatments, which can leverage mate limitation as an additional mechanism for control. More broadly, these results suggest that identifying and setting parasite control targets below densities where Allee effects occur may be more effective and less demanding than higher density control targets. The economics associated with these types of interventions are of interest and will be a critical part of any implementation decision. The results of our model could inform economic approaches, such as that suggested by Liu \& vanhauwaer Bjelland (2014). Agent-based models are particularly amenable to modelling population dynamics at low levels where stochastic processes and 'local conditions' such as mate availability may be influential.

Acknowledgements. We thank the Canada Excellence Research Chair in Aquatic Epidemiology, UPEI, for providing funding to support this study. 


\section{LITERATURE CITED}

<jrn>Aaen SM, Helgesen KO, Bakke MJ, Kaur K, Horsberg TE (2015) Drug resistance in sea lice: a threat to salmonid aquaculture. Trends Parasitol 31:72-81 PubMed doi:10.1016/j.pt.2014.12.006 </jrn>

<jrn>Abolofia J, Asche F, Wilen JE (2017) The cost of lice: Quantifying the impacts of parasitic sea lice on farmed salmon. Mar Resour Econ 32:329-349 doi: $10.1086 / 691981</ j r n>$

<jrn>Aldrin M, Huseby RB, Stien A, Grøntvedt RN, Viljugrein H, Jansen PA (2017) A stagestructured Bayesian hierarchical model for salmon lice populations at individual salmon farms - Estimated from multiple farm data sets. Ecol Model 359:333-348 doi:10.1016/j.ecolmodel.2017.05.019 </jrn>

$<$ jrn>Blanco Gonzalez E, de Boer F (2017) The development of the Norwegian wrasse fishery and the use of wrasses as cleaner fish in the salmon aquaculture industry. Fish Sci 83:661-670 doi:10.1007/s12562-017-1110-4</jrn>

<jrn>Brooker AJ, Papadopoulou A, Gutierrez C, Rey S, Davie A, Migaud H (2018) Sustainable production and use of cleaner fish for the biological control of sea lice: recent advances and current challenges. Vet Rec 183:383 PubMed doi:10.1136/vr.104966</jrn>

<jrn>Cantrell DL, Rees EE, Vanderstitchel R, Grant J, Filgueira R, Revie CW (2018) The use of kernel density estimation with a bio-physical model provides a method to quantify connectivity among salmon farms: spatial planning and management with epidemiological relevance. Front Vet Sci 5:269

$\underline{\text { PubMed }}</$ jrn $>$ doi:10.3389/fvets.2018.00269

<jrn>Connors BM, Lagasse C, Dill LM (2011) What's love got to do with it? Ontogenetic changes in drivers of dispersal in a marine ectoparasite. Behav Ecol 22:588-593 doi:10.1093/beheco/arr024</jrn>

$<$ jrn $>$ Costello MJ (2006) Ecology of sea lice parasitic on farmed and wild fish. Trends Parasitol 22:475-483 PubMed doi:10.1016/j.pt.2006.08.006 </jrn>

$<$ jrn>Costello MJ (2009) The global economic cost of sea lice to the salmonid farming industry. J Fish Dis 32:115-118 PubMed doi:10.1111/j.1365-2761.2008.01011.x </jrn>

<jrn>Cox R, Groner ML, Todd CD, Gettinby G, Patanasatienkul T, Revie CW (2017) Mate limitation in sea lice infesting wild salmon hosts: the influence of parasite sex ratio and aggregation. Ecosphere 8:e02040 doi:10.1002/ecs2.2040</jrn>

<jrn>Dallas TA, Krkošek M, Drake JM (2018) Experimental evidence of a pathogen invasion threshold. R Soc Open Sci 5:171975 PubMed doi:10.1098/rsos.171975</jrn>

<jrn>Ellis T, Turnbull JF, Knowles TG, Lines JA, Auchterlonie NA (2016) Trends during development of Scottish salmon farming: An example of sustainable intensification? Aquaculture 458:82-99 doi:10.1016/j.aquaculture.2016.02.012</jrn>

$<$ jrn> Grimm V, Berger U, Bastiansen F, Eliassen S and others (2006) A standard protocol for describing individual-based and agent-based models. Ecol Model 198:115-126 doi:10.1016/j.ecolmodel.2006.04.023 $</ j r n>$ 
<jrn>Groner ML, Cox R, Gettinby G, Revie CW (2013) Use of agent-based modelling to predict benefits of cleaner fish in controlling sea lice, Lepeophtheirus salmonis, infestations on farmed Atlantic salmon, Salmo salar L. J Fish Dis 36:195-208 PubMed doi: $10.1111 /$ jfd. $12017</$ jrn $>$

<edb>Groner ML, Gettinby G, Stormoen M, Revie CW, Cox R (2014) Modelling the impact of temperature-induced life history plasticity and mate limitation on the epidemic potential of a marine ectoparasite. PLOS ONE 9:e88465</edb> https://doi.org/10.1371/journal.pone.0088465

$<$ jrn>Groner ML, Maynard J, Breyta R, Carnegie RB and others (2016a) Managing marine disease emergencies in an era of rapid change. Philos Trans R Soc Lond B Biol Sci 371:20150364 PubMed doi:10.1098/rstb.2015.0364</jrn>

<jrn>Groner ML, McEwan GF, Rees EE, Gettinby G, Revie CW (2016b) Quantifying the influence of salinity and temperature on the population dynamics of a marine ectoparasite. Can J Fish Aquat Sci 73:1281-1291</jrn> https://doi.org/10.1139/cjfas-2015-0444

<jrn>Imsland AK, Reynolds P, Eliassen G, Hangstad TA, Foss A, Vikingstad E, Elvegård TA (2014) The use of lumpfish (Cyclopterus lumpus L.) to control sea lice (Lepeophtheirus salmonis Krøyer) infestations in intensively farmed Atlantic salmon (Salmo salar L.). Aquaculture 424-425:18-23 doi:10.1016/j.aquaculture.2013.12.033 </jrn>

$<$ jrn>Imsland AK, Reynolds P, Eliassen G, Hangstad TA and others (2015) Feeding preferences of lumpfish (Cyclopterus lumpus L.) maintained in open net-pens with Atlantic salmon (Salmo salar L.). Aquaculture 436:47-51 doi:10.1016/j.aquaculture.2014.10.048 $</$ jrn $>$

<jrn>Imsland AK, Reynolds P, Eliassen G, Mortensen A and others (2016) Is cleaning behaviour in lumpfish (Cyclopterus lumpus) parentally controlled? Aquaculture 459:156165 doi:10.1016/j.aquaculture.2016.03.047</jrn>

<jrn>Imsland AKD, Hanssen A, Nytrø AV, Reynolds P and others (2018) It works! Lumpfish can significantly lower sea lice infestation in large-scale salmon farming. Biol Open 7:bio036301 PubMed doi:10.1242/bio.036301</jrn>

<jrn> Jackson D, Moberg O, Stenevik Djupevåg EM, Kane F, Hareide H (2017) The drivers of sea lice management policies and how best to integrate them into a risk management strategy: An ecosystem approach to sea lice management. J Fish Dis 41:927-933 https://doi.org/10.1111/jfd.12705

<conf>Krkošek M, Morton A, Volpe JP, Lewis MA (2009) Sea lice and salmon population dynamics: effects of exposure time for migratory fish. Proc Biol Sci 276:2819$2828</$ conf $>$ https://doi.org/10.1098/rspb.2009.0317

<jrn>Krkošek M, Connors BM, Morton A, Lewis MA, Dill LM, Hilborn R (2011) Effects of parasites from salmon farms on productivity of wild salmon. Proc Natl Acad Sci USA 108:14700-14704 PubMed doi:10.1073/pnas.1101845108</jrn>

Krkošek M, Connors BM, Lewis MA, Poulin R (2012) Allee effects may slow the spread of parasites in a coastal marine ecosystem. Am Nat 179:401-412</jrn> https://doi.org/10.1086/664458 
<jrn>Lees F, Baillie M, Gettinby G, Revie CW (2008) The efficacy of emamectin benzoate against infestations of Lepeophtheirus salmonis on farmed Atlantic salmon (Salmo salar L.) in Scotland, 2002-2006. PLOS ONE 3:e1549 PubMed doi:10.1371/journal.pone.0001549 </jrn>

$<$ jrn>Liu Y, vanhauwaer Bjelland H (2014) Estimating costs of sea lice control strategy in Norway. Prev Vet Med 117:469-477 PubMed doi:10.1016/j.prevetmed.2014.08.018 $</ j \mathrm{jn}>$

$<$ jrn>Lloyd-Smith JO, Cross PC, Briggs CJ, Daugherty M and others (2005) Should we expect population thresholds for wildlife disease? Trends Ecol Evol 20:511-519 PubMed doi:10.1016/j.tree.2005.07.004</jrn>

<jrn>McEwan GF, Groner ML, Fast MD, Gettinby G, Revie CW (2015) Using agent-based modelling to predict the role of wild refugia in the evolution of resistance of sea lice to chemotherapeutants. PLOS ONE 10:e139128 PubMed doi:10.1371/journal.pone.0139128</jrn>

$<$ jrn>McEwan GF, Groner ML, Burnett DL, Fast MD, Revie CW (2016) Managing aquatic parasites for reduced drug resistance: lessons from the land. J R Soc Interface 13:20160830 PubMed doi:10.1098/rsif.2016.0830</jrn>

<edb>Mortenson S, Skiftesvik AB, Bjelland R, Karlsbakk E, Durif C, Sandlund N (2017) Bruk av rensefisk i lakseoppdrett. In: Svåsand T, Grefsrud ES, Karlsen Ø, Kvamme BO, Glover K, Husa V, Kristiansen TS (eds) Risikorapport norsk fiskeoppdrett. Institute of Marine Science, Bergen, p 161-173</edb>

<edb>Myhre Jensen E, Sevatdal S, Bakke MJ, Kaur K, Horsberg TE (2017) A selection study on a laboratory-designed population of salmon lice (Lepeophtheirus salmonis) using organophosphate and pyrethroid pesticides. PLOS ONE 12:e0178068</edb> https://doi.org/10.1371/journal.pone.0178068

<jrn>Powell A, Treasurer JW, Pooley CL, Keay AJ, Lloyd R, Imsland AK, Garcia de Leaniz C (2017) Use of lumpfish for sea-lice control in salmon farming: challenges and opportunities. Rev Aquacult 10:683-702 https://doi.org/10.1111/raq.12194

Revie CW, Robbins C, Gettinby G, Kelly L, Treasurer JW (2005) A mathematical model of the growth of sea lice, Lepeophtheirus salmonis, populations on farmed Atlantic salmon, Salmo salar L., in Scotland and its use in the assessment of treatment strategies. J Fish Dis 28:603-613</jrn> https://doi.org/10.1111/j.1365-2761.2005.00665.x

$<$ jrn>Rittenhouse MA, Revie CW, Hurford A (2016) A model for sea lice (Lepeophtheirus salmonis) dynamics in a seasonally changing environment. Epidemics 16:8-16 PubMed doi:10.1016/j.epidem.2016.03.003 </jrn>

$<$ conf >Sargent RG (2011) Verification and validation of simulation models. Proc 2011 Winter Simulation Conference, Phoenix, AZ, December 11-14, 2011. IEEE, p 183$198</$ conf $>$

$<\mathrm{jrn}>$ Stephenson JF (2012) The chemical cues of male sea lice Lepeophtheirus salmonis encourage others to move between host Atlantic salmon Salmo salar. J Fish Biol 81:1118-1123 PubMed doi:10.1111/j.1095-8649.2012.03347.x</jrn> 
<jrn>Stien LH, Dempster T, Bui S, Glaropoulos A, Fosseidengen JE, Wright DW, Oppedal F (2016) 'Snorkel' sea lice barrier technology reduces sea lice loads on harvest-sized Atlantic salmon with minimal welfare impacts. Aquaculture 458:29-37 doi:10.1016/j.aquaculture.2016.02.014</jrn>

$<$ jrn>Stormoen M, Skjerve E, Aunsmo A (2013) Modelling salmon lice, Lepeophtheirus salmonis, reproduction on farmed Atlantic salmon, Salmo salar L. J Fish Dis 36:25-33 PubMed doi:10.1111/j.1365-2761.2012.01415.x $</$ jrn $>$

<jrn>Todd CD, Stevenson RJ, Reinardy H, Ritchie MG (2005) Polyandry in the ectoparasitic copepod Lepeophtheirus salmonis despite complex precopulatory and postcopulatory mate-guarding. Mar Ecol Prog Ser 303:225-234 doi:10.3354/meps303225</jrn>

<edb>Treasurer JW, Prickett R, Zietz M, Hempleman C, Garcia de Leaniz C (2018) Cleaner fish rearing and deployment in the UK. In: Treasurer J (ed) Cleaner fish biology and aquaculture applications, 2nd edn. 5M Publishing, Sheffield, p 376-392</edb>

$<$ edb>Wong PP, Losada IJ, Gattuso JP, Hinkel J and others (2014) Coastal systems and lowlying areas. In: Climate Change 2014: Impacts, Adaptation, and Vulnerability. Part A: Global and Sectoral Aspects. Contribution of Working Group II to the Fifth Assessment Report of the Intergovernmental Panel on Climate Change. Cambridge University Press, Cambridge, and New York, NY, p 361-409</edb> 
Table 1. Fixed parameters for all experiments

\begin{tabular}{|l|r|}
\hline Parameter & Value \\
\hline Number of salmon agents & 5000 \\
\hline Lumpfish agent stocking proportion & $10 \%$ \\
\hline Probability of copepodid attachment & 0.7 \\
\hline Background external pressure coefficient & 0.3 \\
\hline Treatment count period & $7 \mathrm{~d}$ \\
\hline Chemical treatment efficacy & Day 154 of the year (early June) \\
\hline Production cycle start & $18 \mathrm{mo}$ \\
\hline Production cycle length & Pall parasitic stages \\
\hline
\end{tabular}

Table 2. Parameters for Expt 1 to investigate the mating requirement. AF: adult female sea lice agents. Multiple values for a parameter indicate variations

\begin{tabular}{|c|c|c|c|}
\hline Parameter & \multicolumn{3}{|c|}{ Value(s) } \\
\hline Temperature & \multicolumn{3}{|c|}{ Norway average (see Table 4) } \\
\hline Treatment threshold (AF per salmon) & \multicolumn{3}{|c|}{0.5} \\
\hline Mating required? & \multicolumn{2}{|c|}{ Yes } & No \\
\hline Lumpfish feeding (daily rate) & none & 0.1 & 0.2 \\
\hline External pressure coefficient ( $C$ in Eq. 2 ) & 1 & 2 & 4 \\
\hline
\end{tabular}

Table 3. Parameters for Expt 2 to investigate external pressure interactions. AF: adult female sea lice agents. Multiple values for a parameter indicate variations

\begin{tabular}{|c|c|c|c|}
\hline Parameter & \multicolumn{3}{|c|}{ Value(s) } \\
\hline Temperature & \multicolumn{3}{|c|}{ Norway average (see Table 4) } \\
\hline Treatment threshold (AF per salmon) & \multicolumn{3}{|c|}{0.5} \\
\hline Mating required? & \multicolumn{3}{|r|}{ Yes } \\
\hline Lumpfish feeding (daily rate) & none & 0.1 & 0.2 \\
\hline External pressure coefficient ( $C$ in Eq. 2$)$ & \begin{tabular}{l|l}
4 &
\end{tabular} & 9 & 19 \\
\hline
\end{tabular}


Table 4. Parameters for Expt 3 to investigate the effects of temperature. AF: adult female sea lice agents. Multiple values for a parameter indicate variations

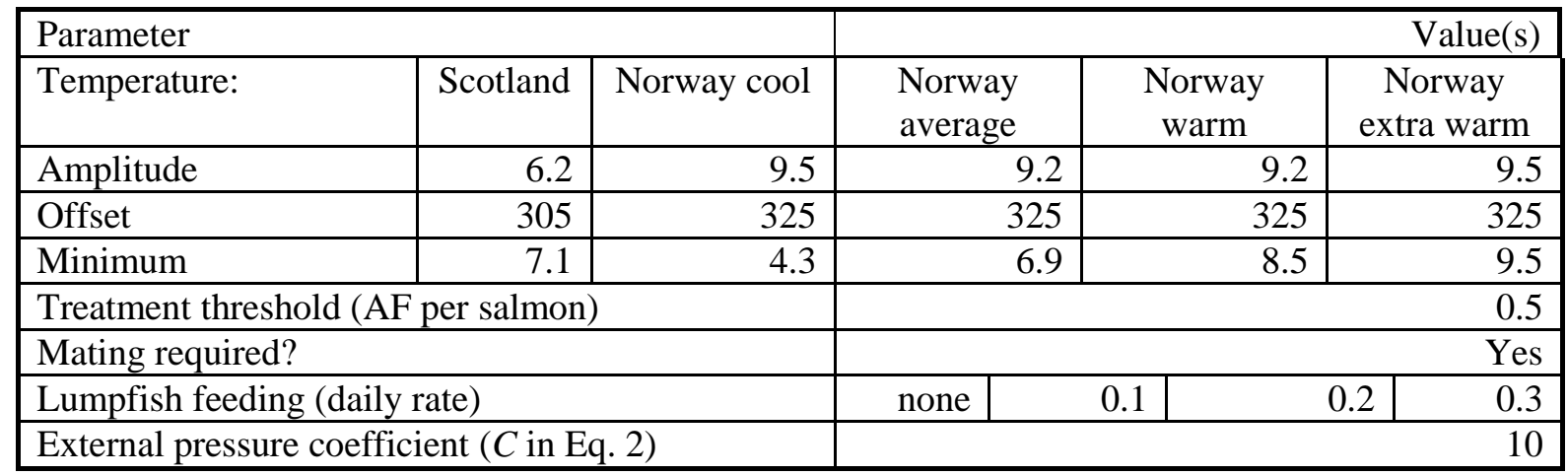

Table 5. Parameters for Expt 4 to investigate interactions with treatment threshold. AF: adult female sea lice agents. Multiple values for a parameter indicate variations

\begin{tabular}{|c|c|c|c|c|}
\hline Parameter & \multicolumn{4}{|c|}{ Value(s) } \\
\hline Temperature & \multicolumn{4}{|c|}{ Norway average (see Table 4) } \\
\hline Treatment threshold (AF per salmon) & 0.1 & & 0.5 & 1 \\
\hline Mating required? & \multicolumn{4}{|r|}{ Yes } \\
\hline Lumpfish feeding (daily rate) & none & 0.1 & & 0.3 \\
\hline External pressure coefficient ( $C$ in Eq. 2 ) & \multicolumn{4}{|r|}{10} \\
\hline
\end{tabular}




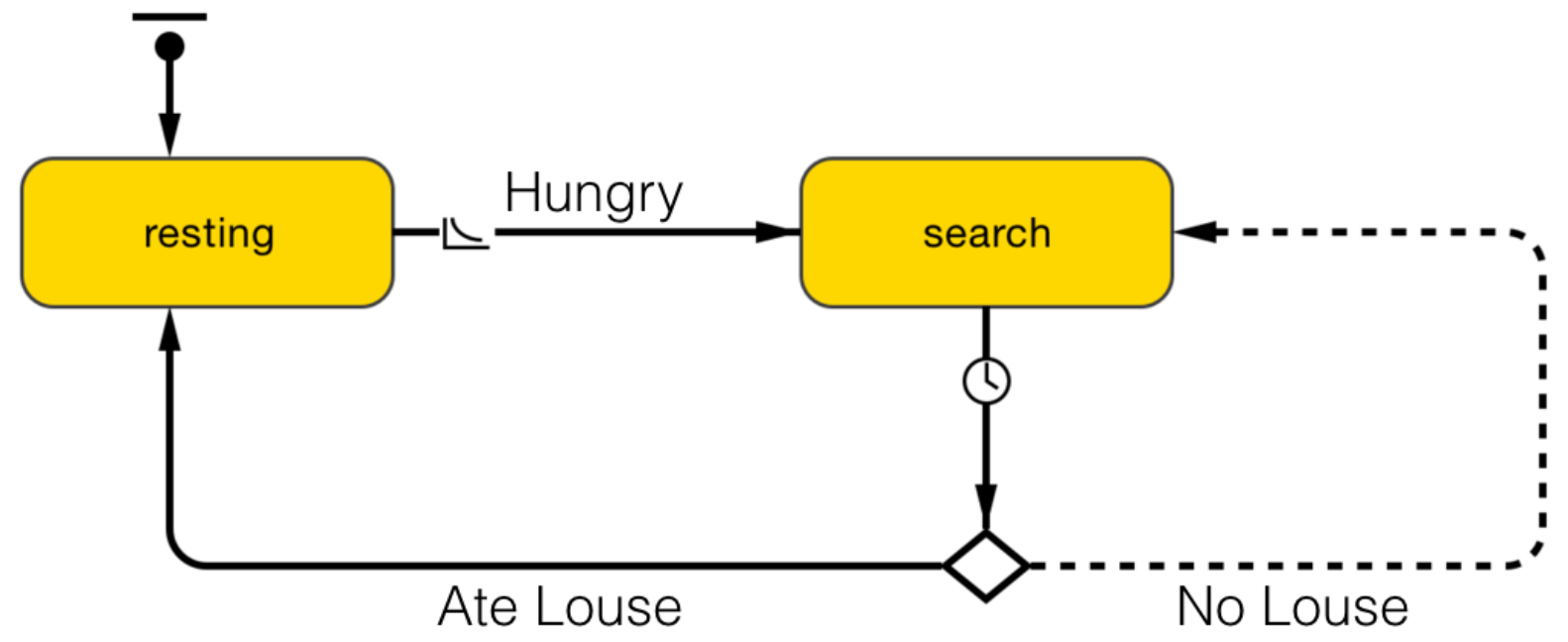

Fig. 1. State transition diagram for lumpfish agents 

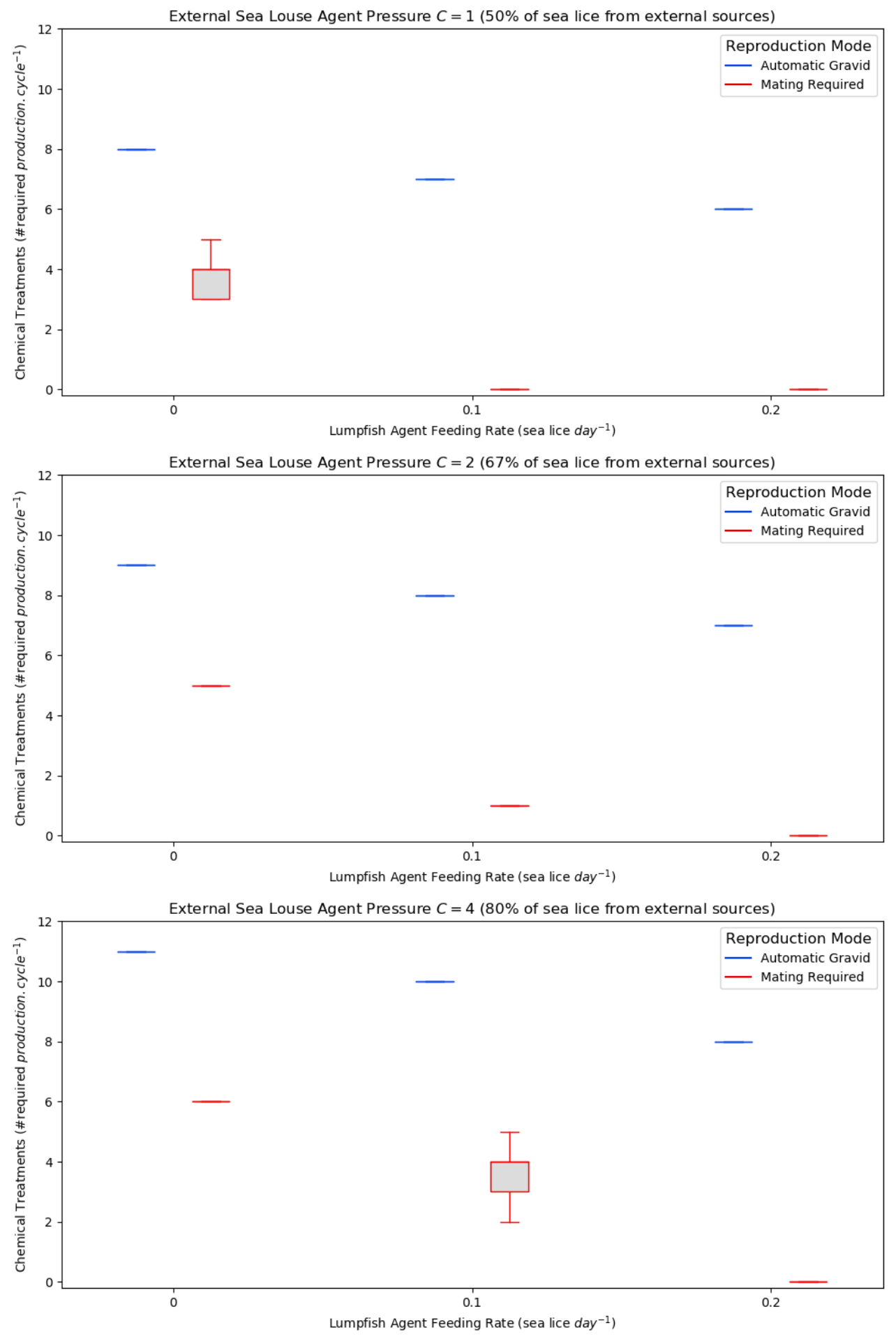

Fig. 2. Total numbers of chemical treatments over a production cycle under varying assumptions as to whether a female sea louse agent must find a mate and based on 3 levels of lumpfish agent feeding, under 3 differing scenarios of internal versus external infestation pressure. All scenarios were replicated 50 times. Box: interquartile range (IQR); whiskers: maximum value $\leq 1.5 \times$ IQR above/below box (outliers not shown). 


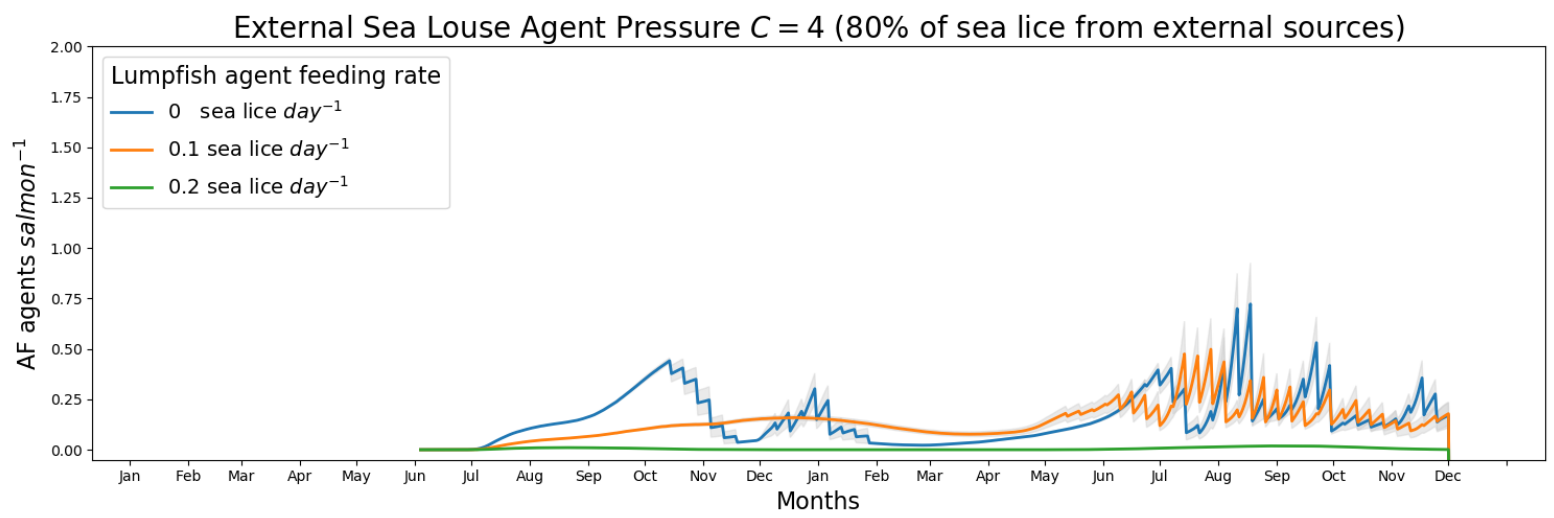

External Sea Louse Agent Pressure $C=9$ ( $90 \%$ of sea lice from external sources)

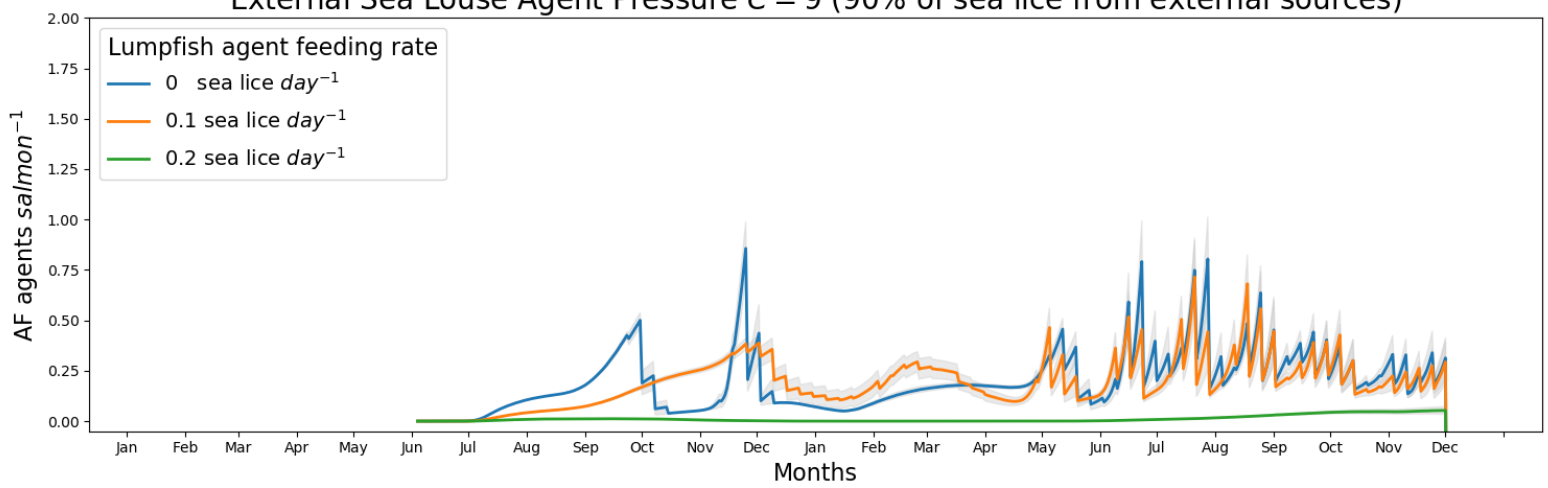

External Sea Louse Agent Pressure $C=19$ (95\% of sea lice from external sources)

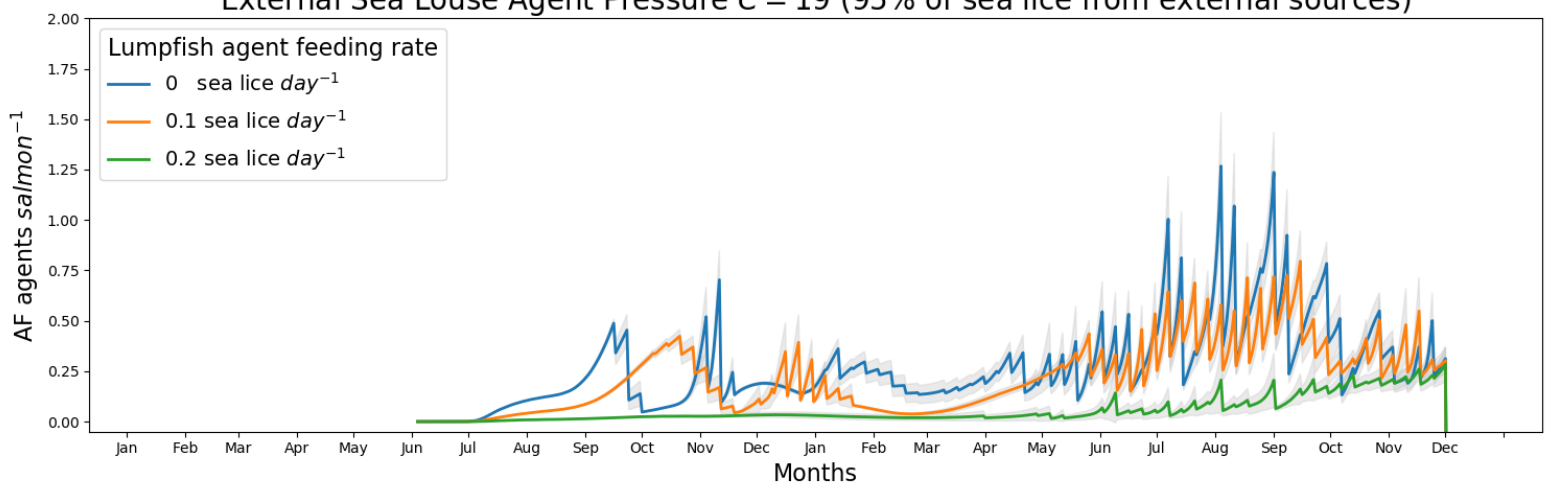

Fig. 3. Dynamics of adult female (AF) sea lice agents over the course of a production cycle based on 3 levels of lumpfish agent feeding, using 3 different scenarios of increasing external infestation pressure. All scenarios were replicated 50 times. Light grey shading: 95\% CI 

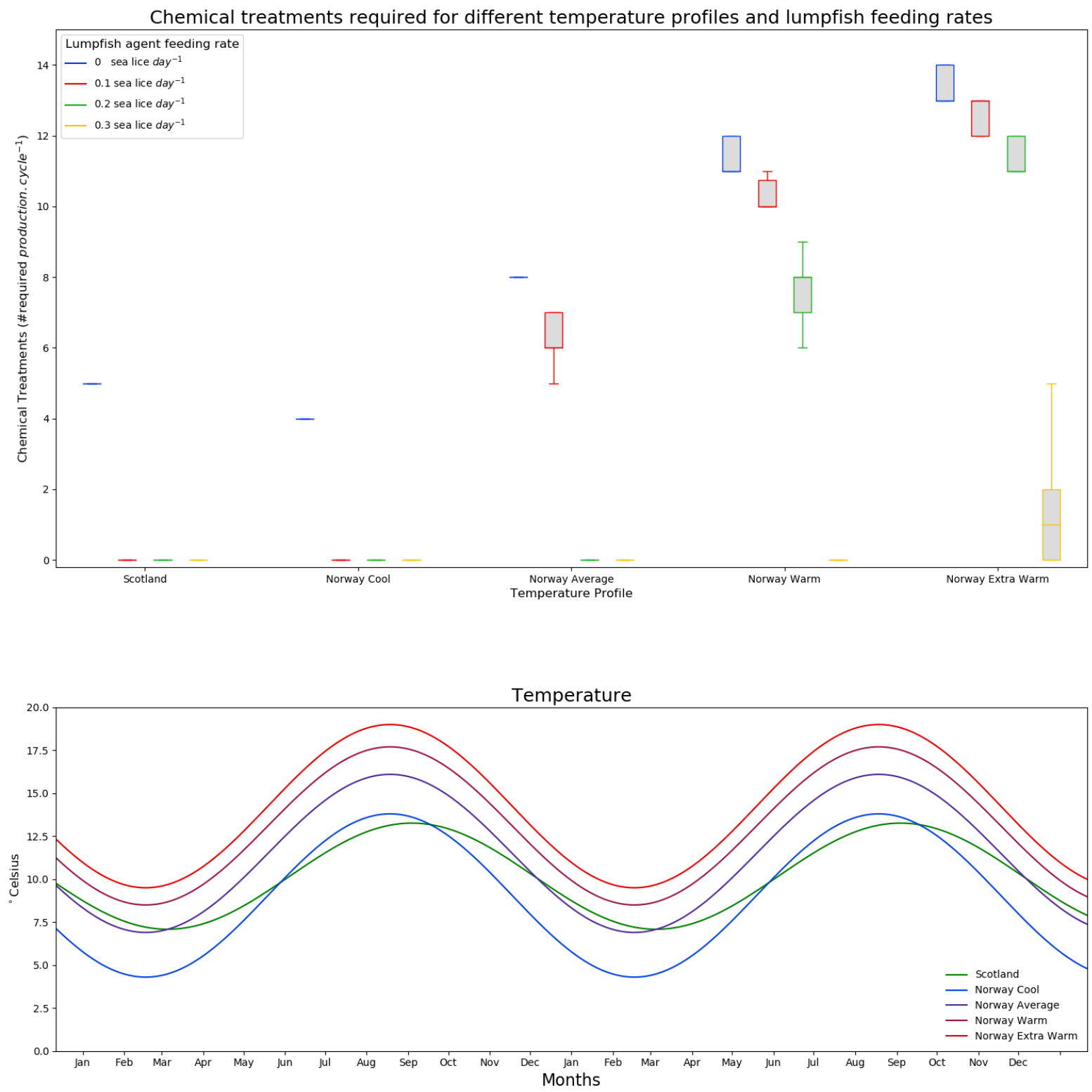

Fig. 4. (Top) Chemical treatments required over a production cycle based on 4 rates of lumpfish agent feeding, with a range of differing seawater temperature scenarios. (Bottom) The 5 temperature profiles used in this study 


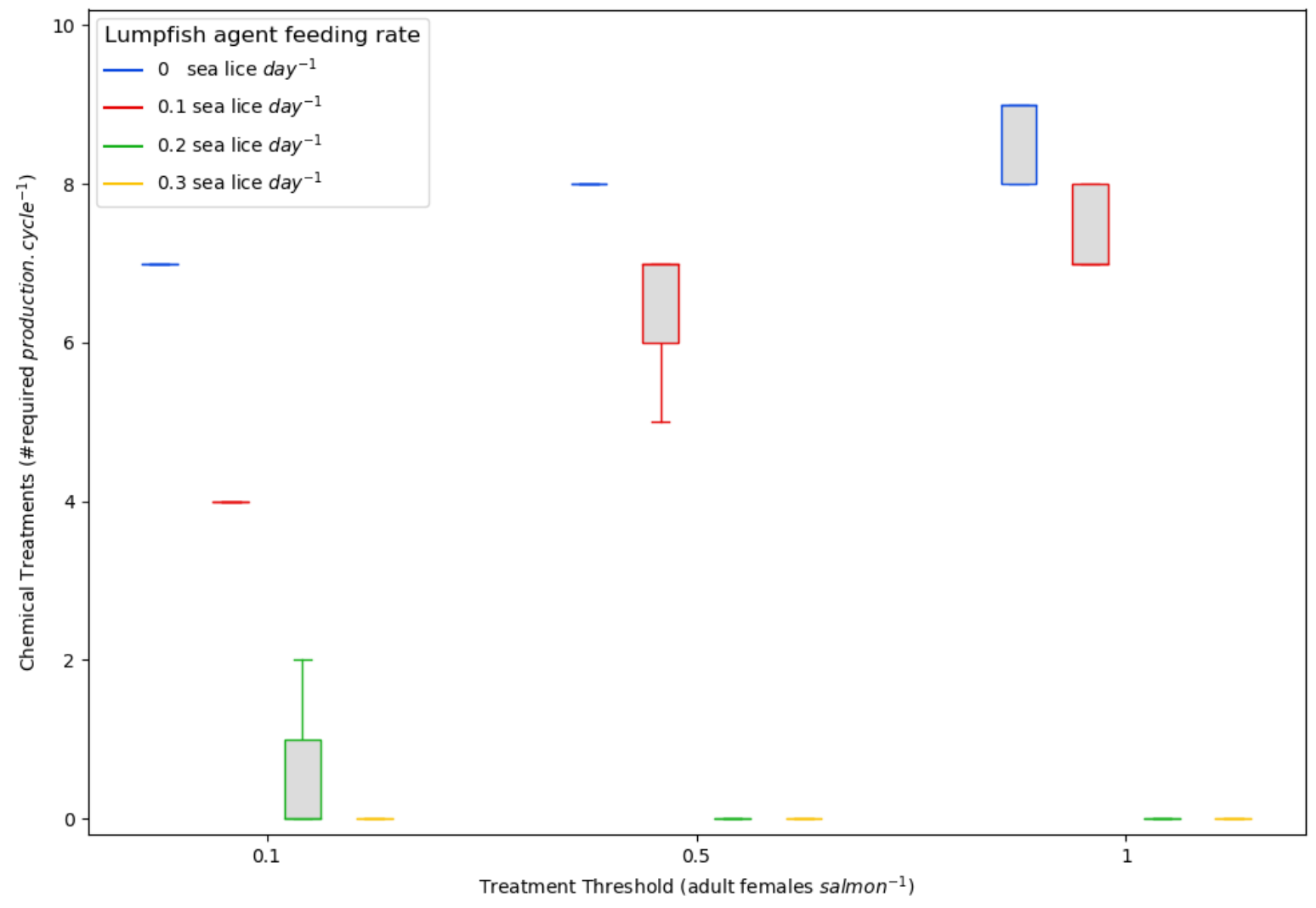

Fig. 5. Chemical treatments required over a production cycle based on 4 levels of lumpfish agent feeding, under the assumption that treatments are applied at 1 of 3 different threshold levels (number of adult female [AF] sea lice agents per salmon). All scenarios were replicated 50 times. Boxplot limits as in Fig. 2 